Postprint of Gielen B., Jordens J., Janssen J., Pfeiffer H., Wevers M., Thomassen L.C.J., Braeken

L., Van Gerven T. (2015). Characterization of stable and transient cavitation bubbles in a milliflow reactor using a MSBL quenching technique. Accepted for publication in Ultrasonics

Sonochemistry.

\title{
Characterization of stable and transient cavitation bubbles in a milliflow reactor using a MBSL quenching technique
}

\author{
B. Gielen ${ }^{1,2}$, J. Jordens ${ }^{1,2}$, J. Janssen ${ }^{1}$, H. Pfeiffer ${ }^{3}$, M. Wevers ${ }^{3}$, L.C.J. Thomassen11,2, L. Braeken ${ }^{1,2}$, T. Van \\ Gerven $^{1}$ \\ ${ }^{1} K U$ Lewven, Department of Chemical Engineering; \\ De Croylaan 46, 3001 Lewven BELGIUM \\ ${ }^{2} \mathrm{KU}$ Lewven, Faculty of Industrial Engineering, $\mathrm{Lab}_{4} \mathrm{U}$; \\ Agoralaan building B box 8, 3590 Diepenbeek BELGIUM \\ ${ }^{3} K U$ Lewven, Department of Metallurgy and Materials Engineering; \\ Kasteelpark. Arenberg 44, 3001 Lewven BELGIUM
}

\begin{abstract}
The bubble type, generated by an ultrasonic field, was studied in a batch and flow reactor using a multibubble sonoluminescence quenching technique with propanol and acetone. The influence of frequency and transducer configuration was evaluated using the same piezo-electric element in both setups. Results show that the bubble type not only depends on the frequency, but also on the power input or transducer configuration. Additionally, the effect of flow on sonoluminescence yield and bubble type was studied in the continuous setup at various frequencies. As the flow becomes turbulent, the sonoluminescence signal reaches a plateau for three out of four frequencies, and a transition from transient to stable cavitation occurs for frequencies below $200 \mathrm{kHz}$.
\end{abstract}

\section{Introduction}

In the last three decades ultrasound has shown an increasing potential for application in processes such as cleaning, emulsification and crystallization.[1-5] The beneficial effects are mostly related to acoustic cavitation, involving the formation and implosion of micron-sized bubbles in less than a few microseconds. As a result, local hot spots are created in which high levels of energy are produced. In aqueous solutions, temperature and pressure can reach levels of $6000 \mathrm{~K}$ and $1000 \mathrm{~atm}$, respectively.[6,7] Under these extreme circumstances, chemical reactivity is promoted through thermolysis, supercritical water oxidation and free radical oxidation. [8,9] In addition, light is emitted from the core of the bubbles during the last stages of the adiabatic collapse, a phenomenon called multibubble sonoluminescence (MBSL).[6,10-12] Probing this light intensity has been used as a tool to determine the bubble type, and indicated that two types of cavitation can be generated; stable and transient bubbles.[12,13] Additional measurements of acoustic emission data, recorded with a hydrophone, confirmed the presence of these two different bubbles types.[14] Transient bubbles only exist for one or a few acoustic cycles, in 
Postprint of Gielen B., Jordens J., Janssen J., Pfeiffer H., Wevers M., Thomassen L.C.J., Braeken

L., Van Gerven T. (2015). Characterization of stable and transient cavitation bubbles in a milliflow reactor using a MSBL quenching technique. Accepted for publication in Ultrasonics

Sonochemistry.

which their radius expands to at least twice the initial size. Due to this limited time frame, it is believed that there is no mass flow of permanent gas through the bubble-liquid interface.[15] This lack of gas cushioning causes transient bubbles to implode very violently, often resulting in the disintegration of smaller bubbles. Stable bubbles, on the other hand, oscillate around their resonance radius for hundreds of acoustic cycles. They contain more gas, and thus have a less violent collapse compared to transient bubbles.[16-18] The generated bubble type is determined by parameters such as host fluid properties, initial bubble size, acoustic pressure and acoustic frequency.[6,19] These factors are described in expressions for cavitation thresholds, like the Blake and Apfel thresholds.[6,19-21] In short, these expressions determine the pressure that the acoustic field needs to generate in order to produce transient bubbles. Additionally, these threshold values form the transition between stable and transient cavitation. Further analysis of the equations shows that the threshold pressures can be more easily overcome at lower frequency, higher acoustic pressure and higher initial bubble size. More transient bubbles are thus expected under these conditions.[22-24]

In the experimental MBSL quenching technique, volatile solutes are added to the aqueous solution. When these solutes evaporate into the cavitation bubbles, they lower the bubble core temperature, and sonoluminescence (SL) quenching - a decrease in SL intensity - occurs.[13,25] The degree of quenching depends on the concentration of the solute at the bubble-solution interface, and not solely on the bulk concentration in the surrounding liquid.[26] Earlier reports demonstrated that by altering the volatility, and the ability of the solute to form hydrogen bonds, one can distinguish between stable and transient cavitation. [10,12,26] For example, Price et al. showed that addition of solutes that do not have the ability to form hydrogen bonds are able to quench SL from transient cavitation.[27] For their experiments with transient cavitation, a pulsed $20 \mathrm{kHz}$ horn operated at 0.1 to $0.4 \mathrm{~W} / \mathrm{cm}^{2}$ was used. At first, a homologous series of methyl ketones (e.g. acetone) was tested at concentrations above $100 \mathrm{mM}$. These solutes were able to quench the SL signal from transient bubbles by more than $40 \%$ compared to the signal of water. Afterwards, a homologous series of $n$-alkyl acetates (e.g. methyl acetate) at the same concentration range was added, and quenching up to $80 \%$ occurred. Although the latter solutes show better quenching, acetone is usually used in the quenching experiments to evidence the presence of transient bubbles. This solute is very volatile and has a high solubility in water of more than $10 \mathrm{M}$, which makes it easy to use in the quenching procedure.[12,27] At solute concentrations below $30 \mathrm{mM}$, both homologous series showed a similar behaviour by which the SL signal first rises before it reaches the 'quenching region' at increasing concentration. This initial rise was higher as the chain length and hydrophobicity of the molecules increases. Price et al. attributed this to a decrease in bubble coalescence. Coalescence increases the bubble size, and these larger bubbles are removed from the ultrasonic field due to buoyancy. Also, these bubbles do not have the correct resonance size to be influenced by the ultrasonic field, and will thus not produce light. Inhibition of coalescence thus yields more SL-active bubbles Moreover, this study showed that hydrophilic volatiles, which can easily form hydrogen bonds, are not able to quench SL signal of transient bubbles. The evaporation of the solute is restricted by the hydrogen bonding, and cannot enter the bubble during its lifetime. An example of this case was studied by 
Postprint of Gielen B., Jordens J., Janssen J., Pfeiffer H., Wevers M., Thomassen L.C.J., Braeken

L., Van Gerven T. (2015). Characterization of stable and transient cavitation bubbles in a milliflow reactor using a MSBL quenching technique. Accepted for publication in Ultrasonics

Sonochemistry.

the addition of a homologous series of alcohols (e.g. propan-1-ol) at concentrations up to $100 \mathrm{mM}$. These compounds can easily form hydrogen bonds and have a volatility that is similar to the earlier tested ketones and acetates. Addition of alcohols with 4 or more carbon atoms in a transient bubble field resulted in an SL enhancement of up to 2-fold compared to the water signal. Solutes with less than 4 carbon atoms did not influence the signal. In short, addition of alcohols in a transient bubble field enhances or does not influence the SL signal. On the contrary, alcohols are able to quench the SL signal when stable bubbles are present. [10,12] Price et al. used a pulsed $515 \mathrm{kHz}$ plate type transducer at 0.1 to $0.4 \mathrm{~W} / \mathrm{cm}^{3}$ to generate these stable bubbles. The fact that quenching is possible during stable cavitation, is related to the life time of these bubbles, assumed to be around 50-200 oscillations. As this is much longer compared to transient cavitation, sufficient evaporation of the alcohols into the bubble occurs so SL quenching can take place.[16] Propanol is often used to perform the experiments as it has a high solubility in water of more than $10 \mathrm{M}$. In addition, its volatility is adequate to use it as a quencher for stable bubbles, whereas lower and more volatile alcohols like ethanol might also affect some transient bubbles.

Previous studies evaluated this quenching technique in batch mode, using different ultrasonic sources, frequencies and volatile substances.[10,12,26] Tronson et al. used a horn-type transducer at $20 \mathrm{kHz}$ with a calorimetric power in the range of 0.09 to $0.2 \mathrm{~W} / \mathrm{cm}^{2}$. They demonstrated that cavitation bubbles were transient in nature, since MBSL was not quenched by addition of aliphatic alcohols like ethanol, propan-1-ol and butan-1-ol in concentrations up to $200 \mathrm{mM}$. Tronson et al. also performed experiments using a $515 \mathrm{kHz}$ plate type transducer with a calorimetric power in the range of 0.04 to $0.4 \mathrm{~W} / \mathrm{cm}^{2}$. It was reported that complete SL quenching was achieved for solutions of $50 \mathrm{mM}$ butanol or $200 \mathrm{mM}$ propanol. Since the alcohols are efficiently quenching the SL signal, it was suggested that the cavitation bubbles were stable in nature at this frequency. Explanations for the difference in bubble type were solely based on the discrepancy in frequency and not on transducer configuration in the reactor, although these greatly differed. These results should thus be interpreted with caution since other studies have emphasized that the reactor design or placement of the transducer in the reactor can strongly influence the ultrasonic field, and therefore also the bubbles.[4,29] Similar experiments with a $20 \mathrm{kHz}$ horn and an acoustic power of $20 \mathrm{~W}$ were conducted by Ashokkumar et al. using propanol concentrations up to $100 \mathrm{mM}$. In the presence of this alcohol, the signal was quenched to about $25 \%$ of its initial value, indicating the presence of mostly transient bubbles. Further experiments conducted at $440 \mathrm{kHz}$ with a plate type transducer show that SL is quenched up to $80 \%$, after which no further quenching was observed despite more addition of propanol. Ashokkumar et al. speculate that this might be due to the existence of $20 \%$ transient cavitation. These findings suggest that both cavitation types are present in a cavitation field, but with a dominating presence of one type. At low frequencies the largest part of the bubbles has a transient character, while at high frequencies stable cavitation is most abundant. However, Ashokkmar et al. showed the dependency of the cavitation type on the ultrasonic source, rather than only on the frequency. Aqueous solutions of propanol up to $100 \mathrm{mM}$ were able to quench the SL signal of a $25 / 37 \mathrm{kHz}$ plate transducer by more than $70 \%$, significantly higher compared to the $20 \mathrm{kHz}$ horn stated earlier. This indicates that low frequency ultrasound, usually classified 
Postprint of Gielen B., Jordens J., Janssen J., Pfeiffer H., Wevers M., Thomassen L.C.J., Braeken

L., Van Gerven T. (2015). Characterization of stable and transient cavitation bubbles in a milliflow reactor using a MSBL quenching technique. Accepted for publication in Ultrasonics

Sonochemistry.

below $50 \mathrm{kHz}$, can also generate stable cavitation when a plate transducer is used. These results show the same trend as the results of Tronson et al., but it is clear that the degree of quenching can differ and strongly depends on the bubble population. A dominant presence of stable cavitation for example can quench the SL signal by using acetone between 0 and $25 \%$, based on the results of these previous studies.

The combination of ultrasound in a confined flow reactor is a novel concept within the chemical industry with high potential for fine chemical and pharmaceuticals production. Placing multiple units in parallel can overcome scale up problems as the reactor dimension remains constant and matches the penetration depth of the ultrasonic wave. In order to fully understand and control the cavitation effects in these reactors, the bubble type and its dependency on flow regime needs to be investigated. The effect of flow or agitation on the sonochemical activity has been studied earlier but yielded conflicting results. Both attenuation and improvement were reported by many authors. [30-36] All explanations for these observations were based on the amount of bubbles, the residence time or mass transfer effects. No correlations were made with the bubble type present in the reactor during the various experimental conditions. Therefore, this study evaluates the cavitation type with a MBSL quenching technique at various flow rates in a rectangular flow cell. To the best of our knowledge, this quenching protocol was never investigated in a continuous millireactor to study the effect of liquid flow on bubble type. Earlier reports, performed in batch reactors, indicate that cavitation type depends on both transducer type and frequency, and that a mix of both stable and transient bubbles is present, with one of these being dominant. However, as different piezo-electric elements were used when comparing frequencies in these reports, it is possible that the frequency effect is caused by a difference in the set-up. Therefore, it is important to evaluate the bubble type using the same ultrasonic actuator operated at different frequencies. This study will thus evaluate also the bubble type using the same piezoelectric element operated at different frequencies and in different setups.

\section{Materials and methods}

\section{Chemicals and experimental procedure}

In all experiments, ultrapure water (Milli-Q, resistance $18.2 \mathrm{M} \Omega . \mathrm{cm}$ ), provided by a SartoriusStedim Arium 611 DI water purification system, was used as the bulk medium. Next, propanol ( $\geq 99.5 \%$ ) and acetone ( $\geq 99.8 \%$ ), purchased from Sigma-Aldrich, are increasingly added to the water up to a concentration of maximum $400 \mathrm{mM}$. The temperature of the solutions was held constant at $20 \pm 2{ }^{\circ} \mathrm{C}$ throughout the experiment. Sonoluminescence signals were recorded at fixed concentration intervals for $30 \mathrm{~s}$ with a gate time of $100 \mathrm{~ms}$, using a photon counting head (Hamamatsu, H11890 series) located on the outside of the setup. Results are shown in this paper on a relative basis compared to the signal of ultrapure water $\left(\mathrm{SL}_{0}\right)$. The results are thus expressed as a ratio with $\mathrm{SL}_{0}$ being the denominator. In all figures, the $\mathrm{SL}_{0}$ value is mentioned so the 
Postprint of Gielen B., Jordens J., Janssen J., Pfeiffer H., Wevers M., Thomassen L.C.J., Braeken

L., Van Gerven T. (2015). Characterization of stable and transient cavitation bubbles in a milliflow reactor using a MSBL quenching technique. Accepted for publication in Ultrasonics

Sonochemistry.

absolute values of all signals can be easily calculated. All experiments were performed three times to allow calculation of the average photon count and standard deviation.

\section{Experimental setup}

Figure 1 shows the experimental reactor setups that were used in this study. To avoid background interference, the complete setup is placed in a dark box. The photon counting head captures the sonoluminescence signal from the active bubbles in the entire reactor volume. Visual inspection showed that these bubbles remained in front of the measuring window at all times, even when flow was applied.
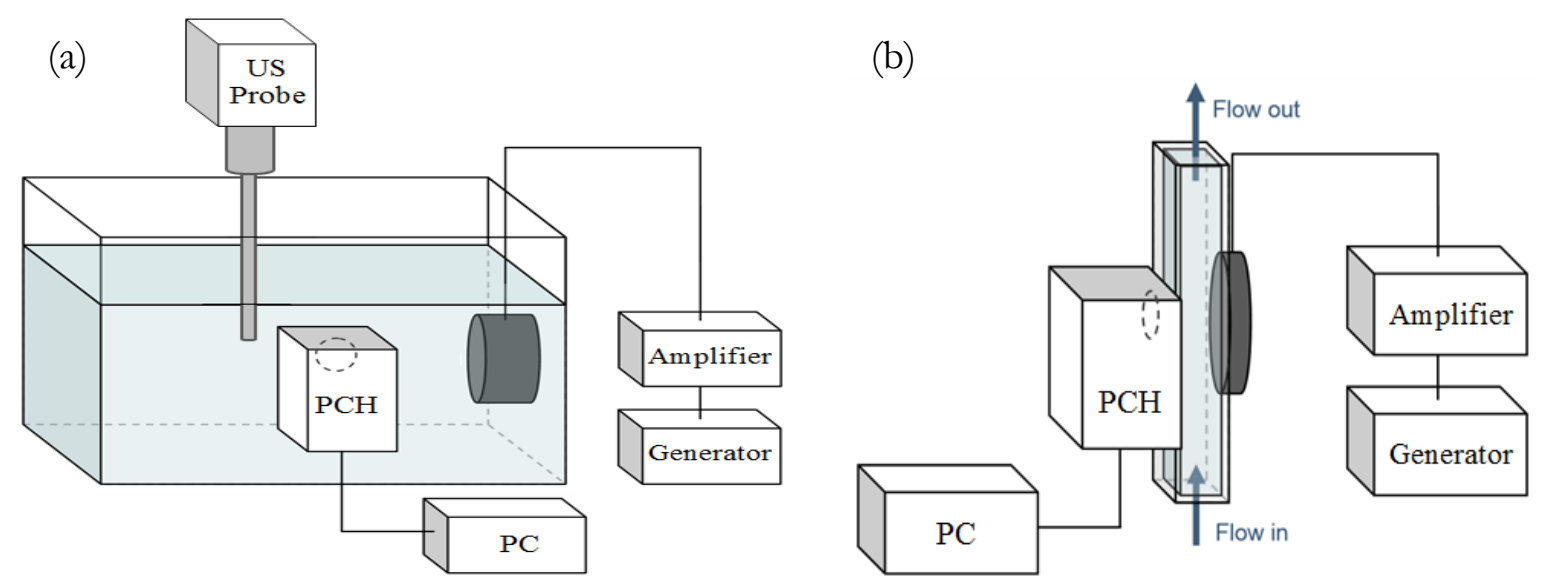

Figure 1: Experimental setups: (a) batch reactor with piezo-electric element submerged in the water as a plate-type transducer, (b) millireactor with piezo-electric element glued to the outer reactor wall. Generator and amplifier are used to create a sinusoidal signal. Photon counting head (PCH) quantifies the sonoluminescence yield.

The batch reactor consists of a rectangular glass tank $(205 \mathrm{~mm} \times 84 \mathrm{~mm} \times 210 \mathrm{~mm})$. Two different ultrasonic sources where used in this reactor. One was a disk transducer (M.P. Interconsulting, type MPI-DISK-SPZT8-500X35) attached to a glass plate and sealed with a plastic cover to prevent contact with water. The transducer was connected to a generator (Picotest G5100) and an amplifier (E\&I 1020L RF). This system operated at frequencies of $46 \mathrm{kHz}$ and $132 \mathrm{kHz}$, with a constant calorimetric power of $12.5 \mathrm{~W}$. The second one is a horn transducer (Hielscher UP200S) with a built-in wave generator and amplifier operating at a resonant frequency of $24 \mathrm{kHz}$. The electrical power was kept constant at $200 \mathrm{~W}$, and a calorimetric power of $93 \mathrm{~W}$ was obtained, corresponding to $38.8 \mathrm{~W} / \mathrm{L}$.

The milliflow reactor consists of a glass cell $(10 \times 10 \times 155 \mathrm{~mm})$. The flow is circulated by a micro centrifugal pump (TCS Micropumps, type M400) and enters the flow cell at the bottom. Flow rates from 0 up to $2 \mathrm{~L} / \mathrm{min}$ were obtained during the experiments. This allows to work in batch conditions, or generate either a laminar or turbulent flow regime. The same generator, amplifier and disk transducer as in the batch setup were used. For this setup, the transducer was glued to the outer wall of the reactor and was able to operate at four resonant frequencies: $46 \mathrm{kHz}$, $119 \mathrm{kHz}, 247 \mathrm{kHz}$ and $564 \mathrm{kHz}$. The calorimetric power was determined at different flow rates 
Postprint of Gielen B., Jordens J., Janssen J., Pfeiffer H., Wevers M., Thomassen L.C.J., Braeken

L., Van Gerven T. (2015). Characterization of stable and transient cavitation bubbles in a milliflow reactor using a MSBL quenching technique. Accepted for publication in Ultrasonics

Sonochemistry.

for all four frequencies, and maintained at $5 \mathrm{~W}$ during the experiments. When only the volume of the flow cell is taken into account, this corresponds to a calorimetric power of $333.3 \mathrm{~W} / \mathrm{L}$.

\section{Results and discussion}

Figure 2 shows the relative quenching data in the presence of propanol and acetone for the $24 \mathrm{kHz}$ probe with a calorimetric power of $93 \mathrm{~W}$, submerged in the batch reactor without any additional flow or agitation. This experiment was performed to deduct a protocol for the interpretation of the quenching data. As was clear from earlier reports, it is not determined which absolute amount of quenching is necessary to conclude that a solute quenches the signal. Interpretation of these results in combination with literature will allow to determine a cut off value that decides whether a solute quenches the signal or not. Overall, propanol is not able to quench sonoluminescence emitted by the $24 \mathrm{kHz}$ probe, suggesting that only transient cavitation is present. The signal in the presence of propanol is even enhanced over the entire concentration region, similar to the results obtained by Price et al., although they only observed the increase in the SL signal for 1-butanol and higher-C alcohols. The rise of SL can be attributed to the surface active properties of the alcohols, hindering the coalescence when they are attached to the bubble surface. This results in a larger amount of cavitation bubbles that contribute to an increased SL intensity. Earlier results by Tronson et al. on the effect of surfactant SDS (sodium dodecyl sulfate) concentration on SL intensity, support these findings. [28] Addition of SDS initially increases the SL signal, followed by a steady value, similar to the propanol curve in Figure 2. They also attribute this effect to the inhibition of bubble coalescence by SDS. Measurement of the coalescence by Lee and Ashokkumar using a capillary setup proved this hypothesis and showed that bubble coalescence decreased in the presence of surfactants like SDS. [7] Therefore, the same effect is expected for alcohols with a terminal hydroxyl group, creating a molecule with a long nonpolar chain and a short polar ending, similar to SDS. Although propanol has a short nonpolar 'tail' compared to the higher alcohols, it is likely that it will influence coalescence in a similar way. The trend of the acetone curve in Figure 2 is identical to the results of Price et al. who were using a $20 \mathrm{kHz}$ horn in combination with a homologous series of ketones. A decrease in bubble coalescence at low acetone concentrations causes the initial rise of SL intensity. At higher acetone concentrations, quenching plays a more significant role and a decrease up to $50 \%$ in the SL intensity is obtained. Measurements at other calorimetric power levels, varying from 17 to $60 \mathrm{~W}$, showed similar results: enhancement by propanol and very limited quenching by acetone. This proves that this horn transducer always generates transient cavitation independent from its power level. Finally, Figure 2 also indicates that the effect of both acetone and propanol remains constant above a concentration of $200 \mathrm{mM}$. The range of this steady state value is comparable to those reported in literature. At this concentration the coalescence inhibition remains constant, so either enhancement or attenuation is possible, depending solely on the ability of the solute to evaporate into the bubble and quench the SL signal. In order to evaluate the bubble type, one should thus check the relative plots of acetone and propanol simultaneously. When only acetone is able to quench the signal transient cavitation is present. If both volatiles are able to quench the 
Postprint of Gielen B., Jordens J., Janssen J., Pfeiffer H., Wevers M., Thomassen L.C.J., Braeken

L., Van Gerven T. (2015). Characterization of stable and transient cavitation bubbles in a milliflow reactor using a MSBL quenching technique. Accepted for publication in Ultrasonics

Sonochemistry.

signal stable bubbles are dominant. Based on own experimental data and that from literature, $50 \%$ quenching or more after addition of propanol is considered as the cut off value for the dominant presence of stable cavitation.[10,12,26] A value below $50 \%$ for propanol will correspond to the presence of transient cavitation with a strong presence of stable bubbles. Enhancement of the signals of both quenchers can always be attributed to a reduced bubble coalescence, increasing the amount of bubbles and boosting the SL yield. [27,28]

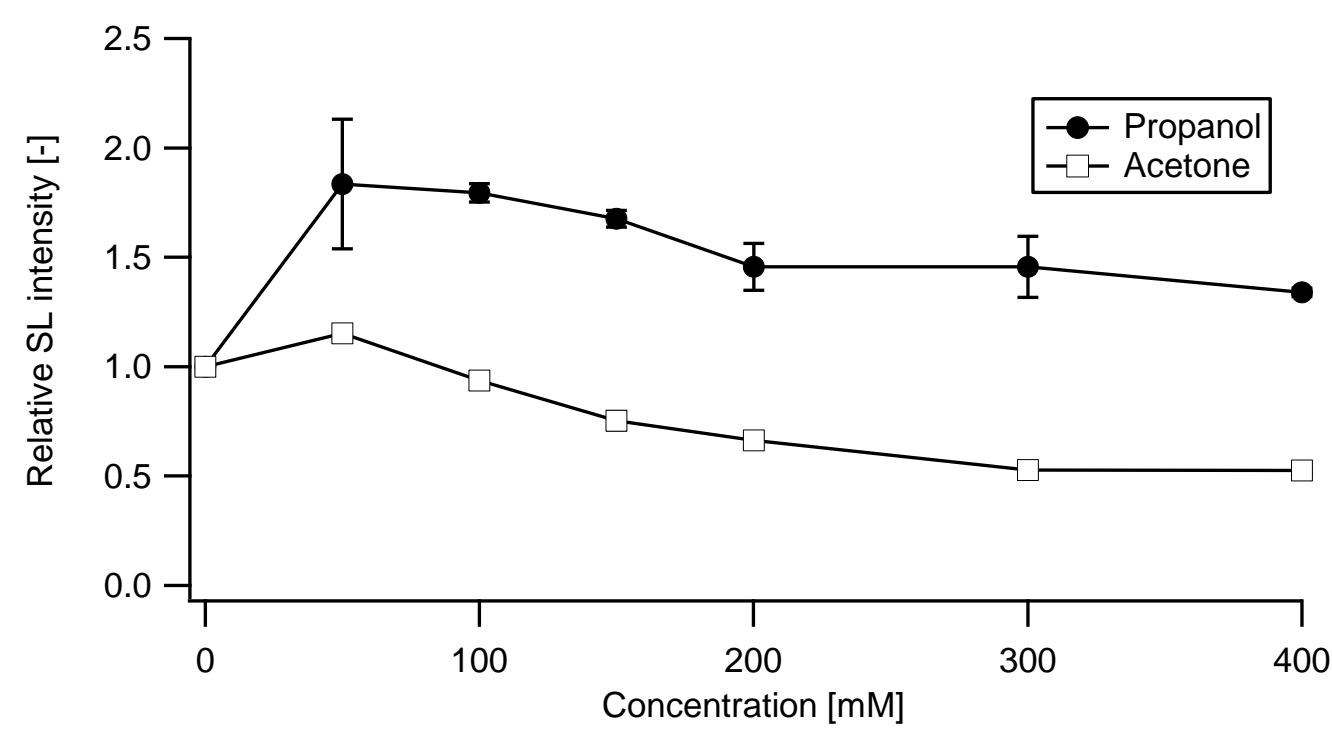

Figure 2: Relative SL quenching data for a $24 \mathrm{kHz}$ horn transducer submerged in the batch reactor without agitation. All measurements are performed three times with the average and standard deviations being displayed. $P_{\text {elec }}=200 \mathrm{~W} ; P_{\text {cal }}=93 \mathrm{~W} . \mathrm{SL}_{0} \approx 83.1$ counts $/ \mathrm{ms}$

Figure 3 and Figure 4 show the SL emission relative to that in ultrapure water at 43 and $46 \mathrm{kHz}$ using the same piezo-electric element in the batch reactor and continuous millireactor, respectively. In both cases, no additional fluid flow or agitation is applied during this set of experiments. The deviations on the measurements in both reactors strongly differ, as can be seen from the error bars that are almost not visible for the batch system, and spread over a large area for the continuous millireactor. This is mostly attributed to the absolute sonoluminescence value of water in both setups that is used to display the signals on a relative basis. In the batch reactor this value fluctuates around 166 counts $/ \mathrm{ms}$, while only 6.6 counts $/ \mathrm{ms}$ are obtained in the millireactor. As this value is placed in the denominator, it is clear that deviations will stand out more in the millireactor as this value is low. Especially the measurements at $50 \mathrm{mM}$ in Figure 4 show high deviations, probably caused by the fluctuations in the bubble coalescence inhibition. At these lower concentrations the inhibition will fluctuate since the equilibrium concentration on the bubble-liquid interface is not reached yet, resulting in a large spread on the amount of bubbles and hence the SL intensity. The rise of the signal at $200 \mathrm{mM}$ propanol is most likely also caused by reduced bubble coalescence resulting in more bubbles compared to the other concentrations. Despite these deviations, it is clear that there are considerable differences between both setups although the same piezo-electric element was used. In the batch reactor 
Postprint of Gielen B., Jordens J., Janssen J., Pfeiffer H., Wevers M., Thomassen L.C.J., Braeken

L., Van Gerven T. (2015). Characterization of stable and transient cavitation bubbles in a milliflow reactor using a MSBL quenching technique. Accepted for publication in Ultrasonics

Sonochemistry.

both propanol and acetone almost completely quench the SL-signal, indicating stable cavitation is dominating. The signal of propanol remains beneath the acetone curve at all times, meaning this compound is quenching more effectively. However, considering the standard deviations of these measurements, it is likely that both acetone and propanol quench the signal to the same extent. In the milliflow reactor, a similar pattern as in Figure 2 arises, with SL enhancement by propanol over the entire concentration region, and an initial improvement followed by a limited reduction of less than $50 \%$ by acetone. Transient cavitation is thus the dominating bubble type in the milliflow system. The difference in cavitation using the same element can be explained in two ways. On the one hand, the reactor geometry is different in both cases, and earlier reports have emphasized that this parameter can strongly influence the ultrasonic field and therefore also the generated cavitation bubbles. $[4,29]$ On the other hand, the calorimetric power expressed per unit of volume or per square $\mathrm{cm}$ is different. For the batch reactor a power of $5.2 \mathrm{~W} / \mathrm{L}$ and $165.5 \mathrm{~mW} / \mathrm{cm}^{2}$ is obtained, for the milliflow cell the power was $333.3 \mathrm{~W} / \mathrm{L}$ and $322.5 \mathrm{~mW} / \mathrm{cm}^{2}$, respectively. As higher power intensity leads to a higher acoustic pressure in the system, the transient cavitation threshold is possibly exceeded in the millireactor, whereas it is not in the batch setup. To determine whether the power or the geometry controls the cavitation type, one should compare these two different setups at the same calorimetric power. In our case, this was not possible, as the transducer was already operated at its maximum within the batch reactor. The possible alternative of a lower power in the milliflow setup resulted in an undetectable SL signal. Therefore, the power levels of these reactors could not be matched [37]

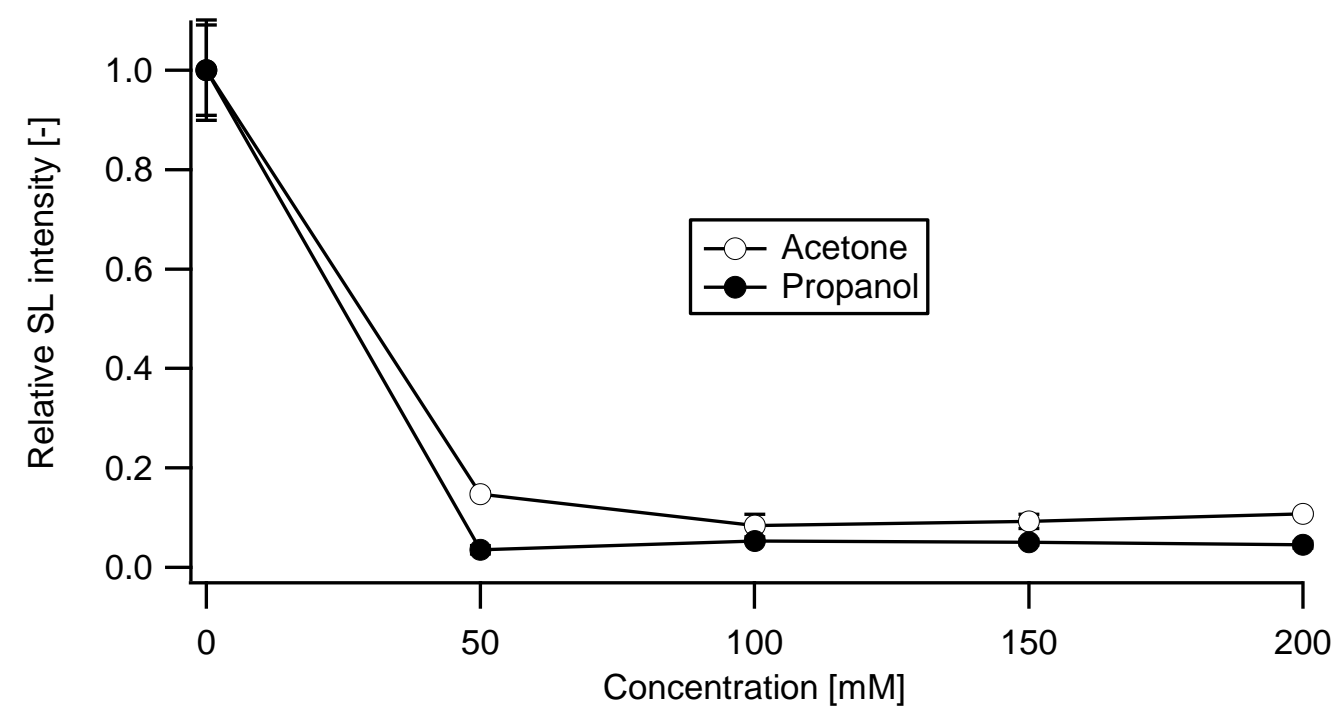

Figure 3: Relative SL quenching data for a piezo-electric element operating at $43 \mathrm{kHz}$, attached to a glass plate, and submerged in a batch reactor without agitation. All measurements are performed three times with the average and standard deviations being displayed. $P_{\text {elec }}=13 \mathrm{~W}, \mathbf{P}_{\text {cal }}=12.5 \mathrm{~W}$. SL $\mathrm{SL}_{0} \approx 166.5$ counts/ms 
Postprint of Gielen B., Jordens J., Janssen J., Pfeiffer H., Wevers M., Thomassen L.C.J., Braeken L., Van Gerven T. (2015). Characterization of stable and transient cavitation bubbles in a milliflow reactor using a MSBL quenching technique. Accepted for publication in Ultrasonics Sonochemistry.

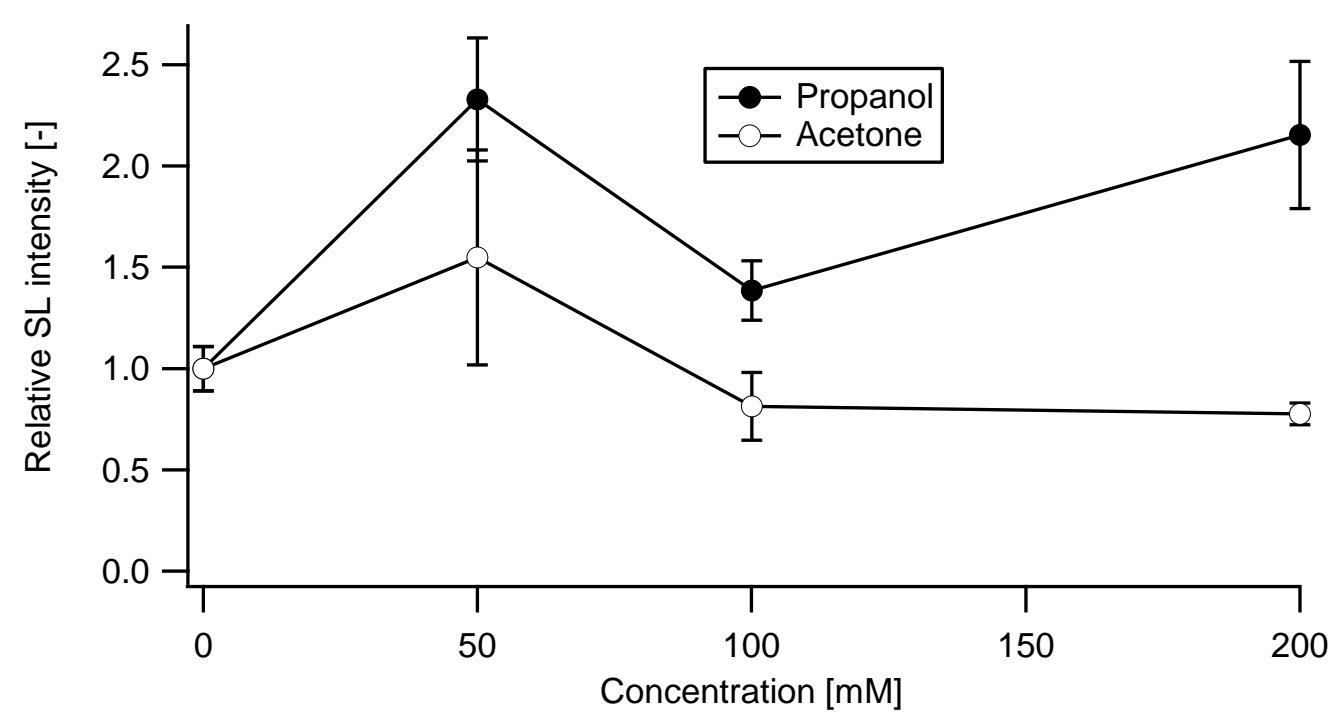

Figure 4: Relative SL quenching data for a piezo-electric element operating at $46 \mathrm{kHz}$, glued onto the outer wall of a continuous millireactor operated without flow. All measurements are performed three times with the average and standard deviations being displayed. $P_{\text {elec }}= \pm 10 \mathrm{~W}, P_{\text {cal }}=5 \mathrm{~W}$. $\mathrm{SL}_{0} \approx 6.6$ counts $/ \mathrm{ms}$

Figure 5 and Figure 6 show the results for the same piezo-electric element operated at 132 and $119 \mathrm{kHz}$ in both a batch and a millireactor, respectively. Similar as at 43 and $46 \mathrm{kHz}$, no flow or agitation was present during these measurements. A similar trend compared to the lower frequency is observed; stable cavitation is present in batch, and transient bubbles arise in the millireactor. From Figure 6 it is clear that propanol does not enhance the signal over the entire concentration region, but limited quenching occurs at $200 \mathrm{mM}$. As stated earlier, this concentration is considered as a steady state value, after which the effect of the quenchers remains constant. Therefore, no further decline of the signal is expected at higher concentrations, and only $25 \%$ SL decrease is obtained. Transient cavitation is thus the dominating bubble type, with a limited amount of stable bubbles present that cause the quenching by propanol at higher concentration. The fact that acetone can quench the signal by more than $50 \%$ confirms that not only transient cavitation is present. 
Postprint of Gielen B., Jordens J., Janssen J., Pfeiffer H., Wevers M., Thomassen L.C.J., Braeken L., Van Gerven T. (2015). Characterization of stable and transient cavitation bubbles in a milliflow reactor using a MSBL quenching technique. Accepted for publication in Ultrasonics Sonochemistry.

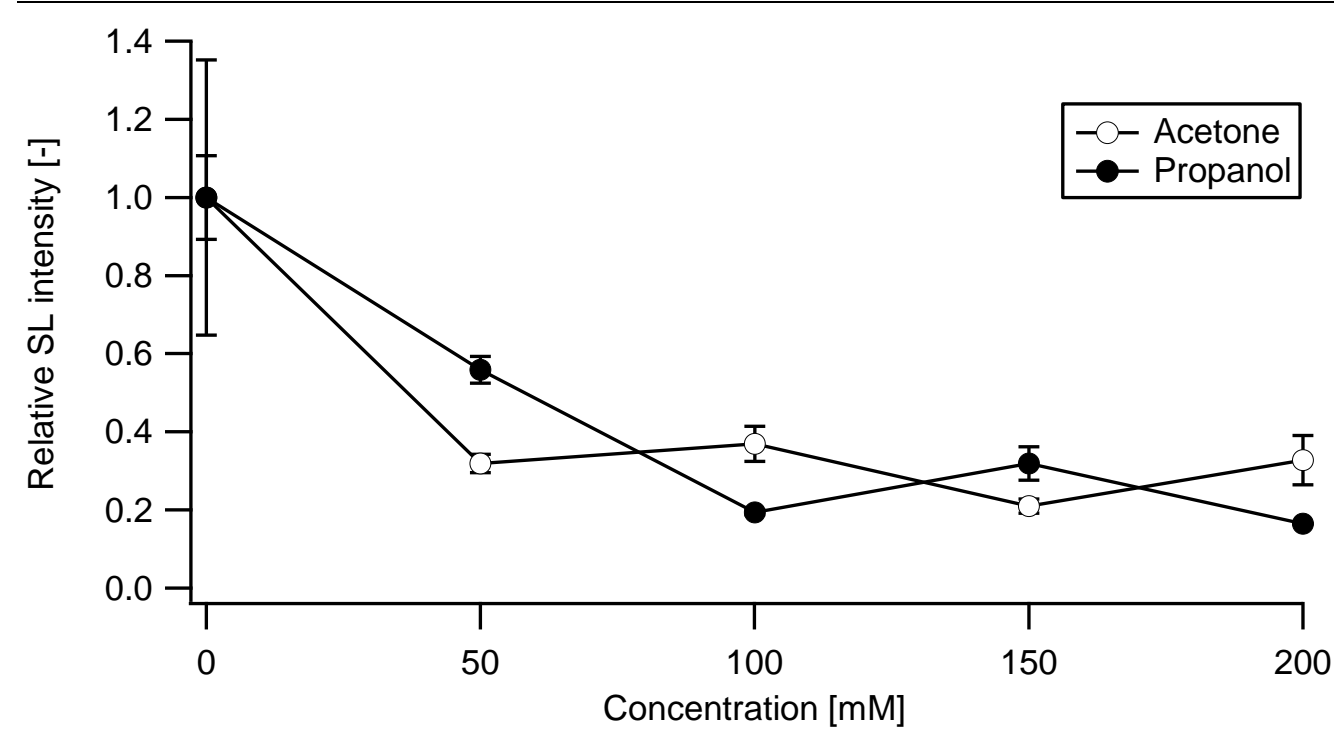

Figure 5: Relative SL quenching data for a piezo-electric element operating at $132 \mathrm{kHz}$, attached to a glass plate, and submerged in a batch reactor without agitation. All measurements are performed three times with the average and standard deviations being displayed. $P_{\text {elec }}=13 \mathrm{~W}, P_{\text {cal }}=12,5 \mathrm{~W} . \mathrm{SL}_{0} \approx 96.8$ counts $/ \mathrm{ms}$

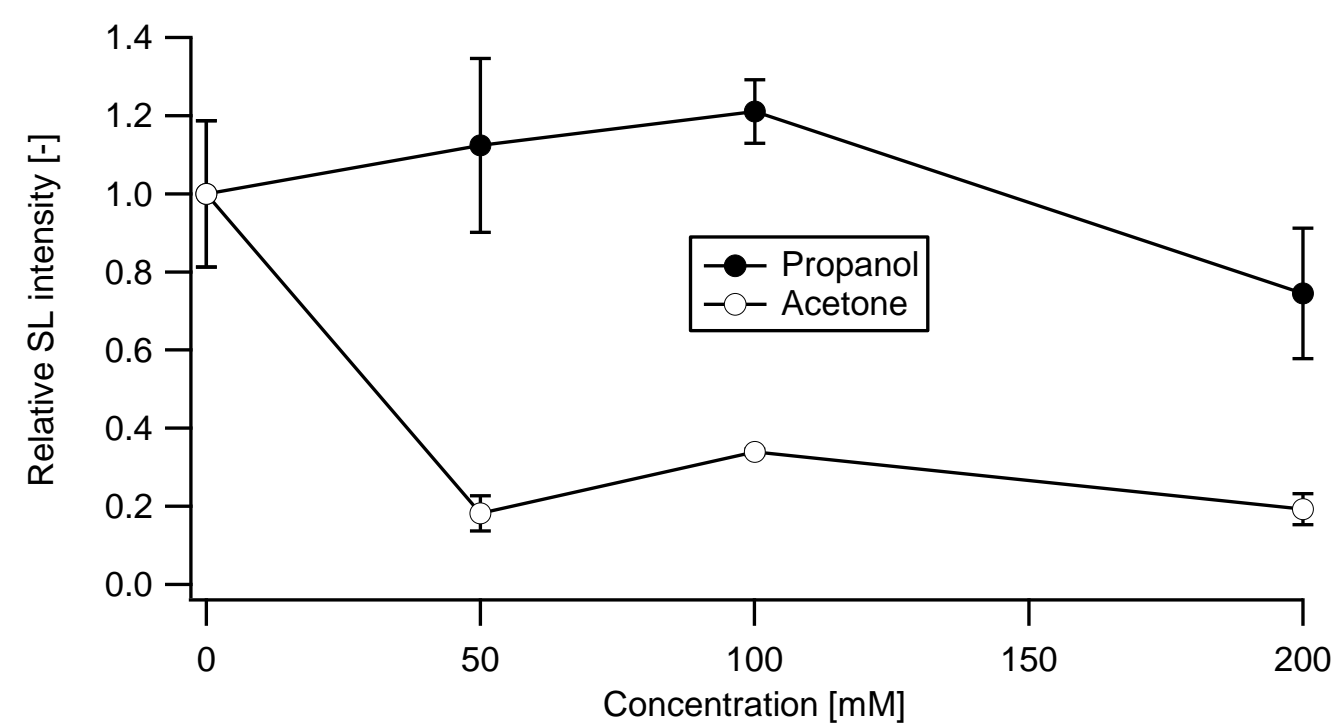

Figure 6: Relative SL quenching data for a piezo-electric element operating at $119 \mathrm{kHz}$, glued onto the outer wall of a continuous millireactor operated without flow. All measurements are performed three times with the average and standard deviations being displayed. $P_{\text {elec }}= \pm 10 \mathrm{~W}, P_{\text {cal }}=5 \mathrm{~W} . \mathrm{SL}_{0} \approx 118.0 \mathrm{counts} / \mathrm{ms}$

When summarizing the above findings, it is shown that low frequency ultrasound does not always generate transient cavitation. Depending on the ultrasonic source, the reactor type and/or input power, also stable bubbles can be generated. Additional experiments in the millireactor without flow, using the piezo electric element at $247 \mathrm{kHz}$ and $564 \mathrm{kHz}$, show that only stable cavitation is obtained. Both acetone and propanol were able to quench the signal by more than $85 \%$ at a concentration of $200 \mathrm{mM}$, corresponding to the plot of stable bubbles. Hence, no transient cavitation occurs although the same power densities are obtained that give rise to these bubble types at the lower frequencies. This can be attributed to the fact that the transient cavitation threshold increases at higher frequencies, so a high power input is needed to change the stable bubbles into transient ones. [6,19] As a conclusion, these results thus show that in some cases 
Postprint of Gielen B., Jordens J., Janssen J., Pfeiffer H., Wevers M., Thomassen L.C.J., Braeken

L., Van Gerven T. (2015). Characterization of stable and transient cavitation bubbles in a milliflow reactor using a MSBL quenching technique. Accepted for publication in Ultrasonics

Sonochemistry.

only the frequency determines the bubble type. However, when the transducer configuration and power intensity changes, the bubble type is not only dependent on frequency.

The effect of flow on bubble type was investigated in the flow reactor at four different frequencies using the quenching technique. Therefore, a SL baseline of ultrapure water at different flow rates was recorded to allow the plots of propanol and acetone to be normalized relative to the signals in water. A relative plot of this baseline, normalized to the signal without any flow, is shown in Figure 7. The flow rate is expressed using the dimensionless Reynolds number $R e$ calculated fora square cross-section with a hydraulic diameter $D_{f}$ of $0.01 \mathrm{~m}$. Furthermore, a density $\rho$ of $998 \mathrm{~kg} / \mathrm{m}^{3}$, a dynamic viscosity of $1 \mathrm{mPa}$.s, and a flowrate $Q$ varying from 0 to almost $3.3 \times 10^{-5} \mathrm{~m}^{3} / \mathrm{s}$ is used. For all frequencies, an enhancement of the sonoluminescence signal was obtained as the flow rate increases. These plots are in accordance with the study of Hatanaka et al. [35] on the effect of fluid flow on MBSL using frequencies from $23-131 \mathrm{kHz}$ and a water circulation of $4 \mathrm{~L} / \mathrm{min}$. SL enhancement was achieved in the presence of flow at various power settings up to $200 \mathrm{~W}$. Additional experiments with a high speed camera elucidated the mechanism, and showed that the fluid flow prevents coalescing and clustering of cavitation bubbles by counteracting the primary and secondary Bjerkness forces. [38] Consequently, this suggests that the bubbles in a flow regime are smaller than in case of a stationary fluid where coalescing and clustering increases the bubble radius. At 46, 247 and $564 \mathrm{kHz}$, a plateau is reached at a Reynolds number of about 2000, close to the end of the laminar region that is ca. 2300 in this reactor [39]. The enhancement can thus be explained by reduced coalescence and clustering up to the point where the flow becomes turbulent. After reaching this flow regime, no further coalescence inhibition occurs, and the amount and size of the of bubbles remains constant. At $119 \mathrm{kHz}$ no plateau is observed, so it is possible that coalescence is prevented over this entire flow regime. This is similar to the empirical results of Thomas who observed that for certain droplet sizes in a turbulent regime no coalescence can occur. [40] Hence, it is possible that at $119 \mathrm{kHz}$ the bubble size matches this critical value and no plateau can be reached since no bubbles are clustering. In addition, a liquid flow always contains small bubbles, up to submicron level, that can serve for nucleation sites of cavitation bubbles.[41] The amount and the size of these bubbles depend on the agitation of the fluid. In a stirred batch reactor, Wu et al. observed that the amount of bubbles increases and the bubble size decreases for higher stirring speeds. By increasing the stirring speed from 500 to $3500 \mathrm{rpm}$, they observed that the average bubble diameter decreases from $800 \mathrm{~nm}$ to $200 \mathrm{~nm}$. Similar results were obtained by Razzaque and his coworkers at increasing flow rates in a continuous reactor. They reported that the initial bubble diameter of about $10 \mathrm{~mm}$ was reduced to less than $3 \mathrm{~mm}$ when the flow velocity increased by 4 times. In addition, more bubbles were detected at these higher flow rates.[42] $\mathrm{A}$ higher amount of bubbles by reduced coalescence, by introduction of submicron nuclei, or a combination of both, means that more nucleation sites are available at higher flow rates. Consequently, more cavitation bubbles that emit light can be formed. 
Postprint of Gielen B., Jordens J., Janssen J., Pfeiffer H., Wevers M., Thomassen L.C.J., Braeken L., Van Gerven T. (2015). Characterization of stable and transient cavitation bubbles in a milliflow reactor using a MSBL quenching technique. Accepted for publication in Ultrasonics

Sonochemistry.
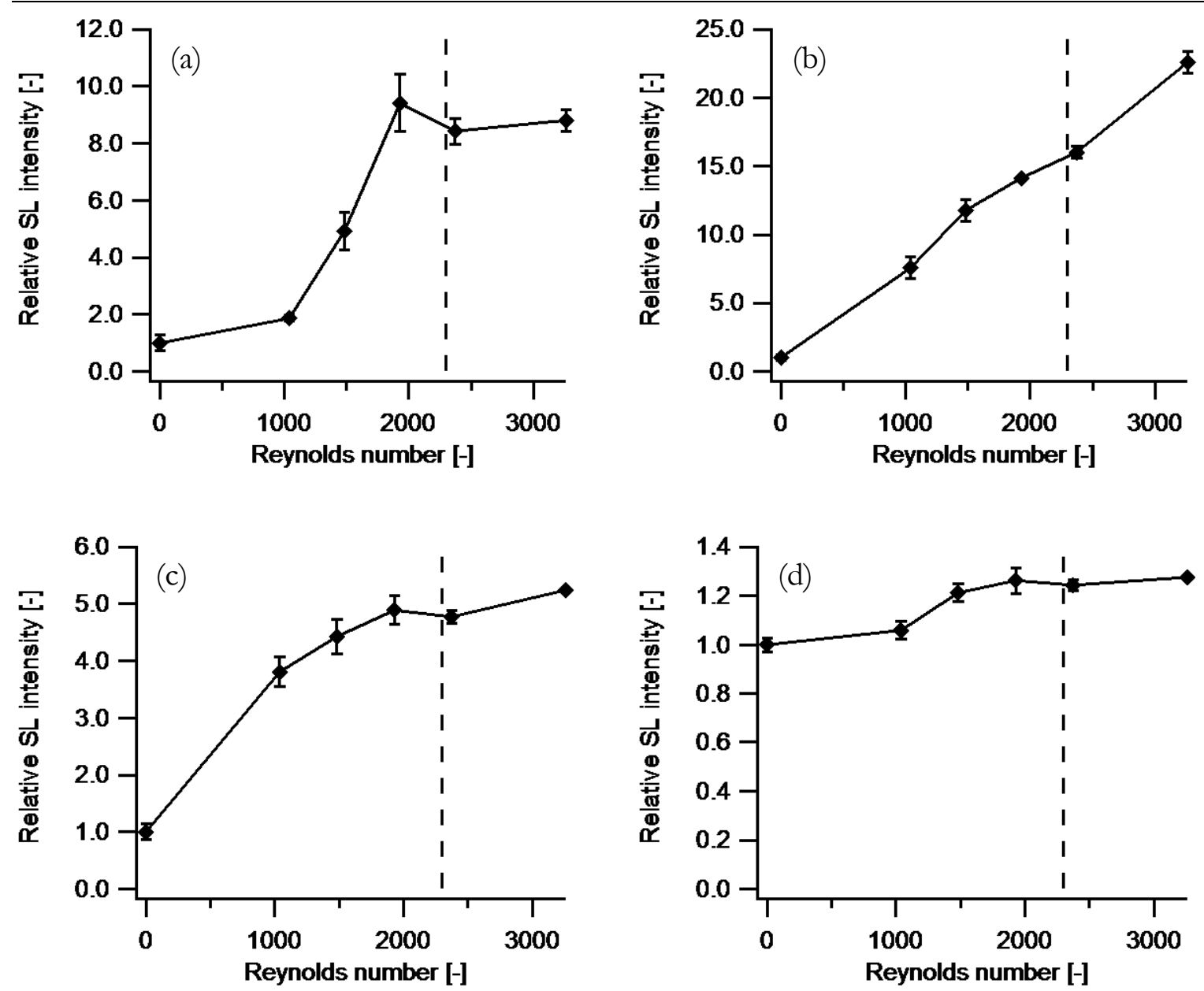

Figure 7: SL intensity of ultrapure water at different flow rates measured at four different frequencies: (a) $46 \mathrm{kHz}$ with $\mathrm{SL}_{0} \approx 23.4$ counts $/ \mathrm{ms}$, (b) $119 \mathrm{kHz}$ with $\mathrm{SL}_{0} \approx 22.3$ counts $/ \mathrm{ms}$, (c) $247 \mathrm{kHz}$ with $\mathrm{SL}_{0} \approx 697.1$ counts $/ \mathrm{ms}$, (d) $564 \mathrm{kHz}$ with $\mathrm{SL}_{0} \approx 3429.7$ counts $/ \mathrm{ms}$. All measurements are performed three times with the average and standard deviations being displayed Calorimetric power was maintained at $5 \mathrm{~W}$ in all conditions.

Figures 8 and 9 show the results for the SL quenching experiments using propanol and acetone at various flow rates for low (46 and $119 \mathrm{kHz})$ and high $(247$ and $564 \mathrm{kHz})$ frequencies, respectively. All signals are displayed relative to the actual SL of ultrapure water at that particular flow rate so only the effect of the quenchers becomes visual as the effect of flow is filtered out. A concentration of $200 \mathrm{mM}$ is used to make sure that the effect on coalescence remains constant, and only the quenching effect is visualized. For the low frequencies of 46 and $119 \mathrm{kHz}$, there is a shift from transient cavitation to stable cavitation as the flow becomes turbulent. In the laminar regime, the propanol plot remains above the $50 \%$ quenching value for both frequencies, indicating that transient bubbles are still present. As the flow becomes turbulent, SL signals of propanol drop below the $50 \%$ value for the two frequencies, indicating that only stable bubbles remain in the reactor. These results are confirmed by evaluation of the acetone curves at both frequencies. Despite an enhancement at $\mathrm{R} e=1000$ for $46 \mathrm{kHz}$, probably due to inhibition of coalescence, acetone is able to quench the signal. This proves that transient bubbles are present in the laminar regime, and the further decline of the signal in the turbulent regime shows that a transition in bubble type occurs. For the higher frequencies, $247 \mathrm{kHz}$ and $564 \mathrm{kHz}$, bubbles are 
Postprint of Gielen B., Jordens J., Janssen J., Pfeiffer H., Wevers M., Thomassen L.C.J., Braeken

L., Van Gerven T. (2015). Characterization of stable and transient cavitation bubbles in a milliflow reactor using a MSBL quenching technique. Accepted for publication in Ultrasonics

Sonochemistry.

stable throughout the entire flow regime as the data points of both quenchers always remain below the $50 \%$. In short, the results in Figures 8 and 9 show that transient bubbles, generated at low frequency, can become stable at high flow rate, while stable bubbles are not altered by flow rate. The threshold for the bubble type transition seems to be around the end of the laminar flow regime, corresponding to a Reynolds number of around 2300.
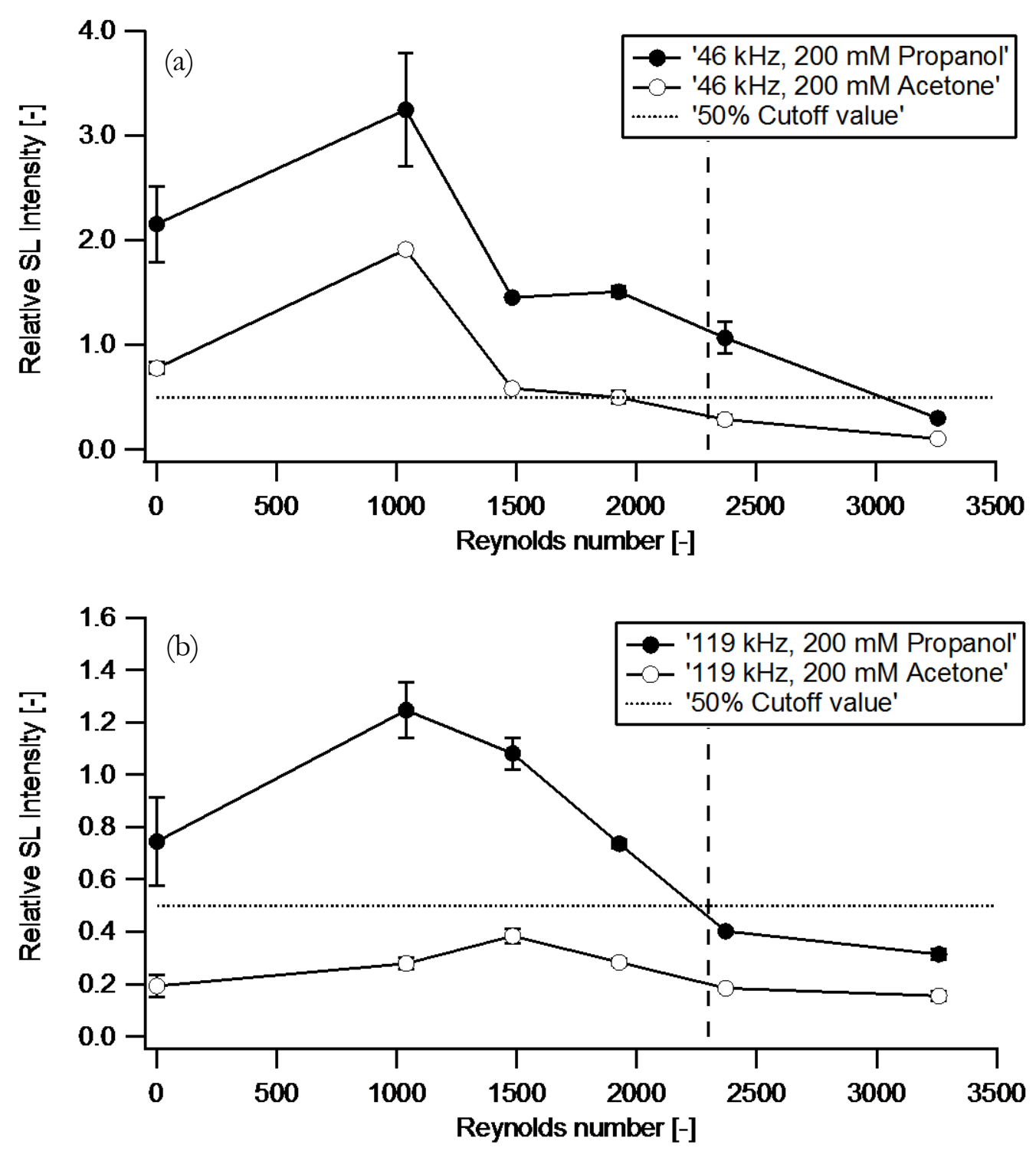

Figure 8: Effect of flow rate on the SL signal for low frequency ultrasound using propanol and acetone. The SL signal of ultrapure water at a particular flow rate is used as the reference for each data point. (a) corresponds to $46 \mathrm{kHz}$ and (b) to $119 \mathrm{kHz}$. All measurements are performed three times with the average and standard deviations being displayed. Calorimetric power was maintained at $5 \mathrm{~W}$ in all conditions. The $50 \%$ cutoff value is displayed with the horizontal dotted line. 
Postprint of Gielen B., Jordens J., Janssen J., Pfeiffer H., Wevers M., Thomassen L.C.J., Braeken L., Van Gerven T. (2015). Characterization of stable and transient cavitation bubbles in a milliflow reactor using a MSBL quenching technique. Accepted for publication in Ultrasonics Sonochemistry.
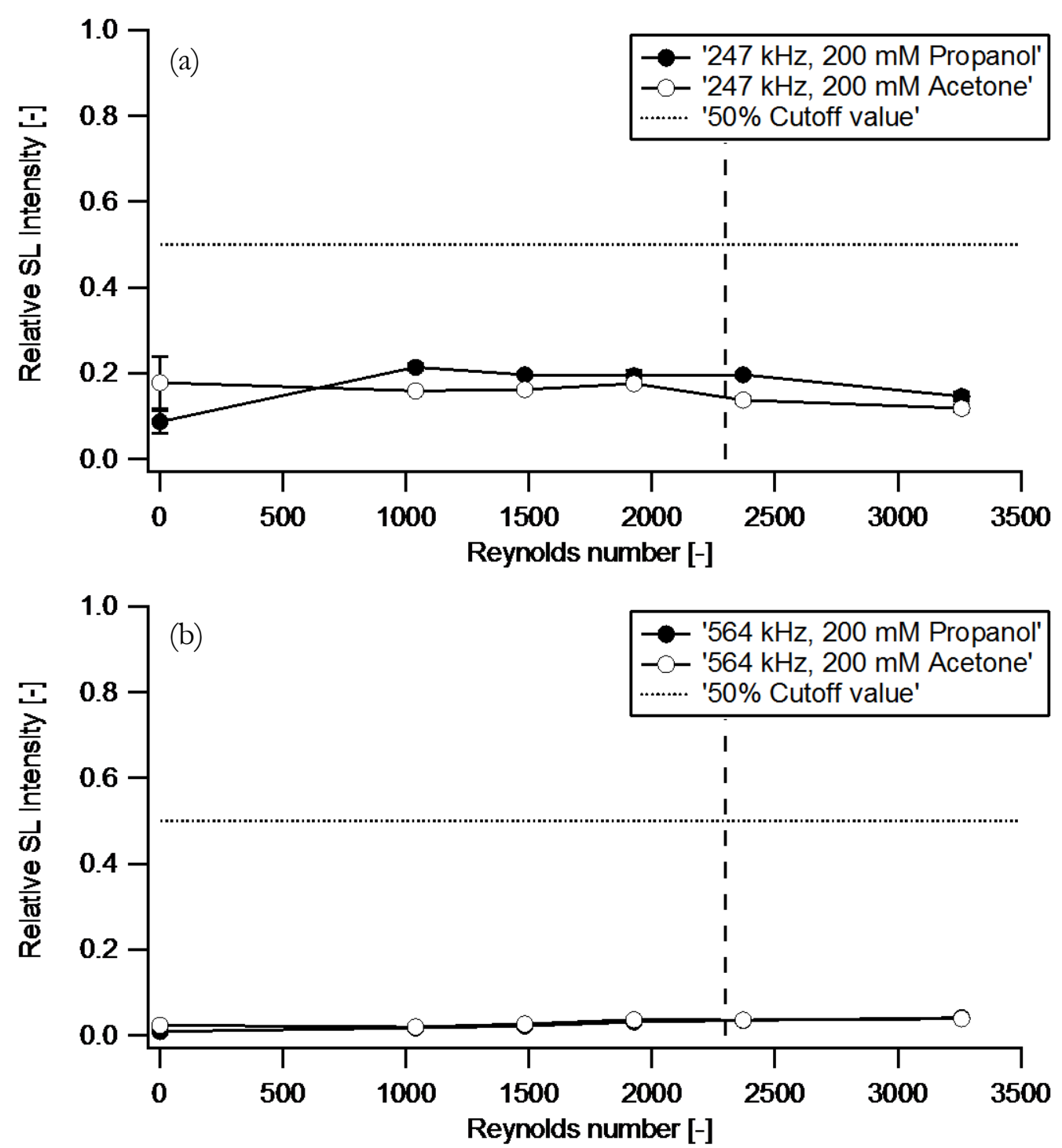

Figure 9: Effect of flow rate on the SL signal for high frequency ultrasound using propanol and acetone. The SL signal of ultrapure water at a particular flow rate is used as the reference for each data point. (a) corresponds to $247 \mathrm{kHz}$ and (b) to $564 \mathrm{kHz}$. All measurements are performed three times with the average and standard deviations being displayed. Calorimetric power was maintained at $5 \mathrm{~W}$ in all conditions. The $50 \%$ cutoff value is displayed with the horizontal dotted line.

A possible explanation for the transition of transient to stable cavitation is related to the size of the bubbles present in the reactor at different flow rates. Flow reduces the bubble size and introduces submicron gas pockets that can act as nuclei. [35,38] Based on the heterogeneous nucleation model of Ramesh et al.[43], formulating that bubble size scales with the size of the nuclei, one can thus assume that smaller cavitation bubbles are present at higher flow rate. Although this model reasons from a solid nucleation surface, it is reasonable to postulate that the same accounts for gaseous nucleation sites that are present during acoustic cavitation. Since stable bubbles have in general a smaller initial radius[19,20], the formation of stable cavitation bubbles might be favoured at high flow rates. 
Postprint of Gielen B., Jordens J., Janssen J., Pfeiffer H., Wevers M., Thomassen L.C.J., Braeken

L., Van Gerven T. (2015). Characterization of stable and transient cavitation bubbles in a milliflow reactor using a MSBL quenching technique. Accepted for publication in Ultrasonics

Sonochemistry.

\section{Conclusion}

This study evaluated the bubble type in a batch and continuous millireactor by using a multibubble sonoluminescence quenching technique with propanol and acetone. To study the effect of frequency and transducer configuration, experiments were performed with the same piezo-electric element operating at a multiple frequencies, but set up in different configurations. Used as a plate type $43 \mathrm{kHz}$ transducer in a batch reactor, stable cavitation was obtained, while transient bubbles arose when the piezo was attached to the outer wall of a flow cell. These observations evidence the fact that stable bubbles can be present at low frequency depending on the power input or transducer configuration in a reactor. The effect of flow was investigated in a rectangular flow cell operated at different frequencies. At turbulent flow rate, the sonoluminescence signal reached a plateau for almost all frequencies, indicating that bubble coalescence was completely inhibited. In addition, a transition from transient to stable cavitation bubbles is observed, at $46 \mathrm{kHz}$ and $119 \mathrm{kHz}$, when the flow becomes turbulent. At frequencies above $200 \mathrm{kHz}$ only stable cavitation occurred independent from the flow rate in this setup. As both bubbles types have different effects, these results indicate the importance of choosing the adequate frequency within the process conditions. This study can thus be valuable for the design and control of ultrasonic reactors for applications that require a specific cavitation type.

\section{Acknowledgements}

The research leading to these results has received funding from the European Community's Seventh Framework Programme (FP7/2007-2013) under grant agreement $n^{\circ}$ NMP2-SL-2012309874 (ALTEREGO). J. Jordens acknowledges funding of a Ph.D. grant by the Agency for Innovation by Science and Technology (IWT).

\section{References}

[1] T. Mason, D. Peters, Practical Sonochemistry: power ultrasound uses and applications, 2nd Editio, Ellis Horwood Publishers, Chichester, 2002.

[2] M. Hauptmann, H. Struyf, S. De Gendt, C. Glorieux, S. Brems, Importance of Bubble Size Control in Ultrasonic Surface Cleaning by Pulsed High-Frequency Sound Fields, ECS J. Solid State Sci. Technol. 3 (2013) N3032-N3040. doi:10.1149/2.007401jss.

[3] B.-K. Kang, M.-S. Kim, J.-G. Park, Effect of dissolved gases in water on acoustic cavitation and bubble growth rate in $0.83 \mathrm{MHz}$ megasonic of interest to wafer cleaning., Ultrason. Sonochem. 21 (2014) 1496-1503. doi:10.1016/j.ultsonch.2014.01.012.

[4] P.R. Gogate, V.S. Sutkar, A.B. Pandit, Sonochemical reactors: Important design and scale up considerations with a special emphasis on heterogeneous systems, Chem. Eng. J. 166 (2011) 1066-1082. doi:10.1016/j.cej.2010.11.069. 
Postprint of Gielen B., Jordens J., Janssen J., Pfeiffer H., Wevers M., Thomassen L.C.J., Braeken L., Van Gerven T. (2015). Characterization of stable and transient cavitation bubbles in a milliflow reactor using a MSBL quenching technique. Accepted for publication in Ultrasonics Sonochemistry.

[5] V.S. Nalajala, V.S. Moholkar, Investigations in the physical mechanism of sonocrystallization., Ultrason. Sonochem. 18 (2011) 345-355. doi:10.1016/j.ultsonch.2010.06.016.

[6] T. Leighton, The Acoustic Bubble, Academic Press, London, 1994.

[7] M. Ashokkumar, The characterization of acoustic cavitation bubbles - an overview., Ultrason. Sonochem. 18 (2011) 864-872. doi:10.1016/j.ultsonch.2010.11.016.

[8] M. a. Beckett, I. Hua, Impact of Ultrasonic Frequency on Aqueous Sonoluminescence and Sonochemistry, J. Phys. Chem. A. 105 (2001) 3796-3802. doi:10.1021/jp003226x.

[9] M.R. Hoffmann, I. Hua, R. Höchemer, Application of ultrasonic irradiation for the degradation of chemical contaminants in water, Ultrason. Sonochem. 3 (1996) S163-S172.

[10] R. Tronson, M. Ashokkumar, F. Grieser, Comparison of the Effects of Water-Soluble Solutes on Multibubble Sonoluminescence Generated in Aqueous Solutions by 20- and 515-kHz Pulsed Ultrasound, J. Phys. Chem. B. 106 (2002) 11064-11068. doi:10.1021/jp020363g.

[11] D. Hammer, L. Frommhold, Sonoluminescence: How bubbles glow, J. Mod. Opt. (2009) 239-277.

[12] M. Ashokkumar, J. Lee, Y. Iida, K. Yasui, T. Kozuka, T. Tuziuti, et al., The detection and control of stable and transient acoustic cavitation bubbles., Phys. Chem. Chem. Phys. 11 (2009) 10118-10121. doi:10.1039/b915715h.

[13] Y.T. Didenko, W.B.M. Iii, K.S. Suslick, Hot Spot Conditions during Cavitation in Water, (1999) 5817-5818.

[14] J. Frohly, S. Labouret, C. Bruneel, I. Looten-Baquet, R. Torguet, Ultrasonic cavitation monitoring by acoustic noise power measurement, J. Acoust. Soc. Am. 108 (2000) 2012 2020.

[15] T. Mason, J.P. Lorimer, Sonochemistry: theory, applications and uses of ultrasound in chemistry, Ellis Horwood Publishers, Chichester, 1988.

[16] D. Sunartio, M. Ashokkumar, F. Grieser, Study of the coalescence of acoustic bubbles as a function of frequency, power, and water-soluble additives, J. Am. Chem. Soc. 129 (2007) 6031-6036. doi:10.1021/ja068980w.

[17] H. Zhao, J.-X. Wang, Q.-A. Wang, J.-F. Chen, J. Yun, Controlled Liquid Antisolvent Precipitation of Hydrophobic Pharmaceutical Nanoparticles in a Microchannel Reactor, Ind. Eng. Chem. Res. 46 (2007) 8229-8235. doi:10.1021/ie070498e.

[18] J.M. Jiju, Application of advanced oxidation processes for the degradation of organic water pollutants, Mahtama Gandhi University, 2000. 
Postprint of Gielen B., Jordens J., Janssen J., Pfeiffer H., Wevers M., Thomassen L.C.J., Braeken L., Van Gerven T. (2015). Characterization of stable and transient cavitation bubbles in a milliflow reactor using a MSBL quenching technique. Accepted for publication in Ultrasonics Sonochemistry.

[19] C.K. Holland, R.E. Apfel, Thresholds for transient cavitation produced by pulsed ultrasound environment, 88 (1990) 2059-2069.

[20] T.. Leighton, Bubble population phenomena in acoustic cavitation, Ultrason. Sonochem. 2 (1995) S123-S136. doi:10.1016/1350-4177(95)00021-W.

[21] A. Harkin, A. Nadim, T.J. Kaper, On acoustic cavitation of slightly subcritical bubbles, Phys. Fluids. 11 (1980) 274-287.

[22] M. Ashokkumar, M. Pankaj, Theoretical and experimental sonochemistry involving inorganic systems, Springer, 2001.

[23] H.G. Flynn, Generation of transient cavities in liquids by microsecond pulses of ultrasound, Acoust. Soc. Am. 72 (1982) 1926-1932.

[24] H.G. Flynn, N. York, C.C. Church, Transient pulsations of small gas bubbles in water, Acoust. Soc. Am. 84 (1988) 985-998.

[25] M. Ashokkumar, P. Mulvaney, F. Grieser, The Effect of $\mathrm{pH}$ on Multibubble Sonoluminescence from Aqueous Solutions Containing Simple Organic Weak Acids and Bases, J. Am. Chem. Soc. (1999) 7355-7359.

[26] M. Ashokkumar, R. Hall, P. Mulvaney, F. Grieser, Sonoluminescence from Aqueous Alcohol and Surfactant Solutions, 5647 (1997) 10845-10850.

[27] G.J. Price, M. Ashokkumar, F. Grieser, Sonoluminescence quenching of organic compounds in aqueous solution: frequency effects and implications for sonochemistry., J. Am. Chem. Soc. 126 (2004) 2755-2762. doi:10.1021/ja0389624.

[28] R. Tronson, M. Ashokkumar, F. Grieser, Multibubble Sonoluminescence from Aqueous Solutions Containing Mixtures of Surface Active Solutes t, J. Phys. Chem. B. 107 (2003) 7307-7311. doi:10.1021/jp034360v.

[29] S. Hatanaka, T. Tuziuti, T. Kozuka, H. Mitome, Dependence of sonoluminescence intensity on the geometrical configuration of a reactor cell., IEEE Trans. Ultrason. Ferroelectr. Freq. Control. 48 (2001) 28-36.

[30] M. Romdhane, A. Gadri, F. Contamine, C. Gourdon, G. Casamatta, Experimental study of the ultrasound attenuation in chemical reactors, Ultrason. Sonochem. 4 (1997) 235-243.

[31] N. Gondrexon, V. Renaudin, C. Petrier, P. Boldo, A. Bernis, Y. Gonthier, Degradation of pentachlorophenol aqueous solutions using a continuous flow ultrasonic reactor: experimental performance and modelling., Ultrason. Sonochem. 5 (1999) 125-131.

[32] B. Yim, H. Okuno, Y. Nagata, Y. Maeda, Sonochemical degradation of chlorinated hydrocarbons using a batch and continuous flow system., J. Hazard. Mater. 81 (2001) 253263. 
Postprint of Gielen B., Jordens J., Janssen J., Pfeiffer H., Wevers M., Thomassen L.C.J., Braeken L., Van Gerven T. (2015). Characterization of stable and transient cavitation bubbles in a milliflow reactor using a MSBL quenching technique. Accepted for publication in Ultrasonics Sonochemistry.

[33] M.J. Bussemaker, D. Zhang, A phenomenological investigation into the opposing effects of fluid flow on sonochemical activity at different frequency and power settings, Ultrason. Sonochem. 21 (2014) 436-445. doi:10.1016/j.ultsonch.2013.07.002.

[34] L. Rong, S. Koda, N. Hiroyasu, Study on the degredation rate constant of chlorobenzene in aqueous solution using a recycle ultrasonic reactor, J. Chem. Eng. Japan. 34 (2001) 1040-1044.

[35] S. Hatanaka, H. Mitome, K. Yasui, S. Hayashi, Multibubble sonoluminescence enhancement by fluid flow., Ultrasonics. 44 (2006) e435-e438.

doi:10.1016/j.ultras.2006.05.022.

[36] Y. Kojima, Y. Asakura, G. Sugiyama, S. Koda, The effects of acoustic flow and mechanical flow on the sonochemical efficiency in a rectangular sonochemical reactor., Ultrason. Sonochem. 17 (2010) 978-984. doi:10.1016/j.ultsonch.2009.11.020.

[37] D. Sunartio, M. Ashokkumar, F. Grieser, The influence of acoustic power on multibubble sonoluminescence in aqueous solution containing organic solutes., J. Phys. Chem. B. 109 (2005) 20044-20050. doi:10.1021/jp052747n.

[38] S. Hatanaka, K. Yasui, T. Kozuka, T. Tuziuti, M. H., Influence of bubble clustering on multibubble sonoluminescence, Ultrasonics. 40 (2002) 655-660.

[39] J.H. Spurk, N. Aksel, Fluid Mechanics, 2nd Editio, Springer-Verlag, Berlin, 2008.

[40] R.M. Thomas, Brief Communication Bubble coalescence in turbulent flows, Int. J. Multiph. Flow. 7 (1981) 709-717.

[41] C. Wu, K. Nesset, J. Masliyah, Z. Xu, Generation and characterization of submicron size bubbles., Adv. Colloid Interface Sci. 179-182 (2012) 123-132. doi:10.1016/j.cis.2012.06.012.

[42] M.M. Razzaque, A. Afacan, S. Liu, K. Nandakumar, J.H. Masliyah, R.S. Sanders, Bubble size in coalescence dominant regime of turbulent air-water flow through horizontal pipes, Int. J. Multiph. Flow. 29 (2003) 1451-1471. doi:10.1016/S0301-9322(03)00123-X.

[43] N.S. Ramesh, D.H. Rasmussen, G.A. Campbell, The heterogeneous nucleation of microcellular foams assisted by the survival of microvoids in polymers containing low glass transition particles. Part I: Mathematical modeling and numerical simulation, Polym. Eng. Sci. 34 (1994) 1685-1697. 